\title{
Influência familiar nas decisões de carreira: análise descritiva em uma amostra
}

\section{brasileira}

\section{Family influence on career decisions: descriptive analysis in a Brazilian sample}

\author{
Jaisso Vautero*, Joana Carneiro Pinto**, Ana Daniela Silva* \\ * Universidade do Minho, **Universidade Católica Portuguesa
}

\begin{abstract}
Resumo
Considerando a influência da família de origem sobre as decisões educacionais e de careira o presente estudo teve por objetivo apresentar estatísticas descritivas de uma escala que avalia a influência familiar nas decisões de carreira em relação a diferentes fatores estruturais e processuais familiares. Participaram 242 estudantes universitários, 181 mulheres $(74.8 \%)$, entre os 21 e 45 anos $(M=25.17 ; D P=5.07)$ de uma universidade do sul do Brasil. Os resultados apontam para diferenças nas médias em alguns fatores estruturais familiares, como o estatuto socioeconómico. Esses resultados devem ser avaliados em estudos posteriores à luz de técnicas estatíticas inferenciais.

Palavras-chave: influência familiar, estudantes
\end{abstract} universitários, fatores processuais, fatores estruturais

\begin{abstract}
Considering the influence of the family of origin on the educational and career decisions, the present study aimed to present descriptive statistics of a scale that evaluates the influence of family in career decisions in relation to familiar structural and processual factors. The sample was composed of 242 university students, 181 women (74.8\%), between 21 and 45 years old $(\mathrm{M}=25.17$; $\mathrm{SD}=$ 5.07) from a university in southern Brazil. The results indicate differences in the means in some family structural factors, such as socioeconomic status. These results should be evaluated in later studies with inferential statistical tests.
\end{abstract}

Keywords: family influence, college students, processual features, structural features.

Pesquisadores do campo da psicologia da educação têm explorado um conjunto de fatores pessoais, interpessoais, e contextuais, associados às decisões educacionais e de carreira. Nestes estudos tem tido particular ênfase a influência que a família de origem exerce sobre a escolha, a transição, a adaptação, a desistência, e a satisfação face a determinado percurso vocacional (e.g.,Dietrich \& Kracke, 2009; Fouad, et al., 2010; Paa \& McWhirter, 2000; Schulenberg, Vondracek, \& Crouter, 1984; Whiston \& Keller, 2004).

Os estudos sobre a influência parental no desenvolvimento vocacional dos filhos têm sido, quase exclusivamente, desenvolvidos com foco na adolescência (e.g., Araújo et al., 2012; Bryant,
Zvonkovic, \& Reynolds, 2006; Faria, Pinto \& Taveira, 2007; Pinto \& Soares, 2000; Otto, 2000; Schultheiss, 2007; Whiston \& Keller, 2004). Deste modo, tem sido negligenciado o impacto da família em fases posteriores do desenvolvimento vocacional, tais como as referentes à transição e adaptação da escola para a universidade e da universidade para o mercado de trabalho (Bardagi, 2007; Pachane, Mercuri, \& Polydoro, 2003). Contudo, com a importância que a frequência do ensino superior adquiriu nos últimos anos, alguns autores defendem a necessidade de se estudar aprofundadamente este novo estádio de desenvolvimento - correspondente ao "jovem adulto" ou "adulto emergente", - situado entre o final da adolescência e o início da idade adulta (Chickering, 1969).

Os jovens adultos, com idades compreendidas entre os 18 e os 25 anos, passam por um conjunto de experiências de transição e de confronto, numa variedade de atividades, papéis e situações (Super, 1976, p.23). A sua resolução bem-sucedida está relacionada com a obtenção de determinados marcos desenvolvimentais, tais como, o desenvolvimento de um sentido de identidade, de vida, de integridade, e de autonomia em direção à interdependência (Chickering, 1969). Nesta fase da vida, à semelhança do que ocorre com os filhos adolescentes, as relações parentais e familiares constituem-se como fatores importantes para a concretização daqueles marcos. Diversos estudos apontam o papel fundamental dos pais enquanto modelos educativos e profissionais, enquanto fontes de apoio, incentivo, informação, e pressão, e enquanto transmissores de motivação, encorajamento, interesses e valores. Quanto maior a percepção, por parte dos filhos, deste apoio parental, maior o planeamento, a integração, o ajustamento, a adaptação, a satisfação e o bem-estar com a sua vida/carreira (Dietrich \& Kracke, 2009; Lent et al., 2002; Porto \& Tamayo, 2006).

A influência familiar no desenvolvimento vocacional tem vindo a ser analisada a partir da ênfase atribuída avariáveis/fatores estruturais e/ou variáveis/fatores processuais (Schulenberg et al., 1984; Pinto \& Soares, 2001; Whiston \& Keller, 2004). As variáveis estruturais incluem, entre outras, o estatuto socioeconómico, a educação parental e a situação profissional parental. Por sua vez, as variáveis processuais incluem, entre outras, 
o apoio, o encorajamento e as expectativas parentais, os padrões de comunicação e interação pais-filhos,e os estilos educativos parentais.

O impacto destas variáveis no desenvolvimento vocacional é complexo e dinâmico. E, embora pareçam existir evidências de que as últimas têm um papel mais determinante nas aspirações, no planeamento, na tomada de decisão e na resolução de problemas relativos à carreira, existe uma grande necessidade de sistematizar e aprofundar o conhecimento que possuíamos acerca da participação dos pais (e de outros familiares) enquanto figuras de referência no âmbito do desenvolvimento de carreira dos seus filhos jovens adultos (Guerra \& Braungart-Rieker, 1999; Lankard, 1995; Otto, 2000; Ribeiro, 2005).

O presente estudo é um trabalho exploratório a respeito da influência familiar na implementação de decisões de carreira em jovens universitários. Tem por objetivo apresentar estatísticas descritivas de uma escala que avalia a influência familiar nas decisões de careira, em relação a diferentes fatores estruturais e processuais familiares.

\section{Método}

\section{Participantes}

Participaram no estudo 242 estudantes universitários, 181 mulheres $(74.8 \%)$, entre os 21 e 45 anos $(M=$ $25.17 ; D P=5.07)$.

\section{Instrumentos}

Foram utilizados: (i) o formulário sociodemográfico para recolher dados sobre as variáveis estruturais familiares (e.g., idade, sexo, idade da mãe no nascimento do filho, ordem de nascimento, situação profissional e habilitações literárias parentais, composição do agregado familiar, curso universitário e existência de inscrição prévia noutra licenciatura); e, (ii) a versão em português brasileiro da Escala de Influência da Família, desenvolvida por Fouad e colaboradores (2010), que identifica o grau em que os membros da família influenciam a tomada de decisões de carreira. É composta por 22 itens organizados quatro subescalas: o apoio informacional (e.g., "A minha família compartilhou informação comigo sobre como conseguir um emprego"); o apoio financeiro (e.g., "Se eu passasse por uma situação difícil carreira, minha família iria me apoiar financeiramente"); as expectativas (e.g., "A minha família espera que a minha escolha da carreira reflita os seus desejos"); e, os valores e as crenças (e.g., "A minha família espera que a minha carreira corresponda a nossas crenças/valores"). Utilizando uma escala tipo Likert de 6 pontos $(1=$ discordo totalmente e $6=$ concordo totalmente), foi solicitado aos participantes que indicassem $o$ grau de concordância com as afirmações apresentadas sobre a influência familiar no seu desenvolvimento educacional e de carreira.

$\mathrm{O}$ prestígio social da licenciatura e o estatuto socioeconómico foram variáveis adicionais utilizadas. $\mathrm{O}$ prestígio social da licenciatura foi obtido a partir de indicadores oficiais acerca do salário médio da ocupação correspondente à licenciatura e do rácio entre os candidatos e as vagas disponíveis naquela licenciatura. Estas variáveis foram agrupadas através de uma análise de agrupamento hierárquico (hierarquical cluster analysis), resultando em dois grupos, de alto e baixo prestígio. $\mathrm{O}$ estatuto socioeconómico foi através do índice de Hollingshead (1975), com base na estrutura do agregado familiar, habilitações literárias e profissão parental.

\section{Procedimentos de recolha e análise de dados}

Os participantes foram recrutados entre estudantes universitários de uma universidade localizada no sul do Brasil, com ingresso entre 2012 e 2013. Foi enviado um convite de participação por email com um link para o Surveymonkey (http://www.surveymonkey.com). Foram enviados 2054 convites, retornando 303 formulários, dos quais 242 válidos.

Todas as análises foram realizadas utilizando o Software Statistical Package for the Social Sciences for Windows (SPSS), versão 20.0.

Tabela 1.

Estatística descritiva para a Escala de Influência da Família por idade de ingresso na universidade e sexo

\begin{tabular}{lccccc}
\multicolumn{6}{l}{ Estatistica descritiva para a Escala de Influência da Família por idade de ingresso na universidade e sexo } \\
Variável & \multicolumn{7}{c}{$\begin{array}{c}\text { Apoio } \\
\text { informacional } \\
M(D P)\end{array}$} & $\begin{array}{c}\text { Apoio financeiro } \\
M(D P)\end{array}$ & $\begin{array}{c}\text { Expectativas } \\
M(D P)\end{array}$ & $\begin{array}{c}\text { Valores e } \\
\text { crenças } \\
M(D P)\end{array}$ \\
\hline $\begin{array}{l}\text { Sexo } \\
\text { Feminino }\end{array}$ & $181(74.8)$ & $25.47(7.03)$ & $17.8(4.36)$ & $14.83(4.69)$ & $6.65(2.95)$ \\
$\quad$ Masculino & $61(25.2)$ & $25.68(6.54)$ & $17.9(4.26)$ & $14.8(4.91)$ & $6.56(2.62)$
\end{tabular}

Idade de ingresso na universidade

\begin{tabular}{lccccc}
$<19$ & $112(46.3)$ & $25.58(6.76)$ & $18,04(3,96)$ & $14.54(4.49)$ & $6.65(2.69)$ \\
$19-24$ & $105(43.4)$ & $24.9(6.88)$ & $18.07(4.50)$ & $14.7(4.80)$ & $6.34(2.98)$ \\
$25-35$ & $21(8.7)$ & $26.14(8.09)$ & $15.81(4.33)$ & $15.9(4.88)$ & $7.62(2.60)$ \\
$>35$ & $4(1.6)$ & $27.25(6.93)$ & $16(7.62)$ & $20.25(6.70)$ & $8.25(5,12)$ \\
\hline
\end{tabular}


Tabela 2.

Estatística descritiva para a Escala de Influência da Família por composição do agregado familiar, ordem de nascimento e idade da mãe no nascimento do filho

\begin{tabular}{|c|c|c|c|c|c|}
\hline Variável & $n(\%)$ & $\begin{array}{c}\text { Apoio } \\
\text { informacional } \\
M(D P)\end{array}$ & $\begin{array}{c}\text { Apoio financeiro } \\
M(D P)\end{array}$ & $\begin{array}{c}\text { Expectativas } \\
M(D P)\end{array}$ & $\begin{array}{c}\text { Valores e } \\
\text { crenças } M(D P)\end{array}$ \\
\hline \multicolumn{6}{|l|}{$\begin{array}{l}\text { Composição do agregado } \\
\text { familiar }\end{array}$} \\
\hline Conjugal & $140(59.1)$ & $25.22(7.01)$ & $18.06(4.25)$ & $14.47(4.6)$ & $6.42(2.92)$ \\
\hline Monoparental Feminina & $75(31.6)$ & $25.98(6.46)$ & $17.88(4.25)$ & $15.10(4.77)$ & $6.93(2.72)$ \\
\hline Monoparental Masculina & $3(1.3)$ & $29.67(8.96)$ & $19.00(1.00)$ & $12.00(6.56)$ & $5.50(2.89)$ \\
\hline $\begin{array}{l}\text { Não referiu família de } \\
\text { origem }\end{array}$ & $13(5.5)$ & $20.77(5.58)$ & $16.38(4.54)$ & $14.77(4.73)$ & $7.31(2.69)$ \\
\hline $\begin{array}{l}\text { Cuidado por outros } \\
\text { familiares que não os pais }\end{array}$ & $6(2.5)$ & $23.33(9.00)$ & $17.5(4.76)$ & $16.33(4.03)$ & $5.17(1.47)$ \\
\hline \multicolumn{6}{|l|}{ Ordem de nascimento } \\
\hline Primogénito & $200(82.6)$ & $25.24(7.03)$ & $17.80(4.23)$ & $14.88(4.79)$ & $6.74(2.89)$ \\
\hline Não primogénito & $42(17.3)$ & $25.89(7.03)$ & $17.98(4.79)$ & $14.55(4.47)$ & $6.09(2.78)$ \\
\hline \multicolumn{6}{|l|}{$\begin{array}{l}\text { Idade da mãe no nascimento } \\
\text { do filho }\end{array}$} \\
\hline$<19$ & $7(3.3)$ & $22.29(7.76)$ & $18.00(1.83)$ & $11.43(2.99)$ & $4.86(3.29)$ \\
\hline $19-24$ & $57(27.3)$ & $25.38(7.40)$ & $17.74(4.24)$ & $15.21(4.63)$ & $6.48(3.01)$ \\
\hline $25-35$ & $129(61.7)$ & $25.20(6.48)$ & $17.93(4.44)$ & $14.50(4.63)$ & $6.65(2.81)$ \\
\hline$>35$ & $16(7.6)$ & $28.29(6.83)$ & $18.63(3.88)$ & $16.27(4.70)$ & $7.06(2.60)$ \\
\hline
\end{tabular}

\section{Resultados}

Os resultados mostram que a generalidade dos participantes são mulheres $(74,8 \%)$, com ingresso mais frequente na universidade antes dos 19 anos, ainda que um expressivo número tenha ingressado entre 19 e 24 anos (cf. tabela 1). A configuração do agregado familiar é predominantemente conjugal (nuclear), a segunda composição mais frequente é a monoparental feminina. Quanto à ordem de nascimento, $82.6 \%$ da mostra é composta de primogénitos $(n=200)$, e as mães tinham entre 25 e 35 anos no nascimento do estudante em $61.7 \%$ dos casos (cf. tabela 2). $\quad$ Em relação à situação ocupacional dos pais, a situação de empregado é mais frequente, seguido de profissional liberal empresário em nome individual, tanto para o pai como para a mãe (cf. tabela 3 ). O estatuto socioeconómico de nível 3 , ou mediano, representa $58.6 \%$ dos casos, não existindo participantes com pontuação que os classificasse no nível mais alto do sistema utilizado (acima de 55 pontos) (cf. tabela 5). Relativamente ao prestígio social da licenciatura, apenas $15 \%$ dos estudantes frequentavam cursos codificados como de alto prestígio (cf. tabela 4). 
Tabela 3.

Estatística descritiva para a Escala de Influência da Família por área situação ocupacional dos pais

\begin{tabular}{|c|c|c|c|c|c|}
\hline Variável & $n(\%)$ & $\begin{array}{c}\text { Apoio } \\
\text { informacional } M \\
(D P) \\
\end{array}$ & $\begin{array}{c}\text { Apoio financeiro } \\
M(D P)\end{array}$ & $\begin{array}{c}\text { Expectativas } \\
M(D P) \\
\end{array}$ & $\begin{array}{c}\text { Valores e } \\
\text { crenças } M(D P)\end{array}$ \\
\hline \multicolumn{6}{|c|}{ Situação ocupacional mãe } \\
\hline Empregada & $78(41.7)$ & $25.3(7.12)$ & $18.08(4.29)$ & $14.96(4.75)$ & $6.58(2.86)$ \\
\hline $\begin{array}{l}\text { Profissional liberal ou } \\
\text { empresário em nome } \\
\text { individual }\end{array}$ & $41(21.9)$ & $25.59(6.96)$ & $18.41(3.62)$ & $14.68(4.94)$ & $6.50(3.25)$ \\
\hline Subsídio público & $11(5.9)$ & $24.77(6.98)$ & $16.91(4.35)$ & $15.77(4.62)$ & $7.00(2.41)$ \\
\hline Desempregada & $3(1.6)$ & $25.75(4.72)$ & $17.67(2.31)$ & $14.67(4.16)$ & $6.33(3.05)$ \\
\hline Retirada & $28(15.0)$ & $26.19(7.01)$ & $17.93(4.39)$ & $14.41(4.86)$ & $6.76(2.21)$ \\
\hline Trabalho doméstico & $26(13.9)$ & $24.14(6.60)$ & $17.23(4.55)$ & $14.69(4.95)$ & $6.15(3.11)$ \\
\hline \multicolumn{6}{|l|}{ Situação ocupacional pai } \\
\hline Empregado & $65(41.4)$ & $25.81(6.69)$ & $17.95(4.35)$ & $13.92(4.57)$ & $6.45(3.04)$ \\
\hline $\begin{array}{l}\text { Profissional liberal ou } \\
\text { empresário em nome } \\
\text { individual }\end{array}$ & $55(35.0)$ & $23.89(7.22)$ & $17.67(4.12)$ & $15.26(4.85)$ & $6.35(1.70)$ \\
\hline Subsídio público & $11(7.0)$ & $26.58(7.28)$ & $19.00(3.00)$ & $15.82(4.56)$ & $6.69(1.70)$ \\
\hline Desempregado & $14(8.9)$ & $23.8(7.50)$ & $17.36(5.60)$ & $12.86(4.33)$ & $6.36(3.73)$ \\
\hline Retirado & $12(7.6)$ & $26(6.95)$ & $17.92(4.81)$ & $12.25(4.00)$ & $5.54(1.98)$ \\
\hline
\end{tabular}

A leitura das tabelas 3 e 4 indica que, em relação à Escala de Influência da Família, as mães que se encontram em situação de reforma, de desempregada ou de profissional liberal em nome individual, e os pais que se encontram em situação de subsídio público e reforma são os que apresentam níveis mais elevados de apoio informacional; as mães que se encontram em situação de empregada ou profissional liberal e os pais que se encontram em situação de subsídio público são os que apresentam níveis mais elevados de apoio financeiro; as mães e pais em situação de subsídio público são os que apresentam expectativas mais elevadas, assim como valores e crenças.
Tabela 4.

Estatística descritiva para a Escala de Influência da Família por estatuto socieconómico familiar e prestígio social do curso

\begin{tabular}{cccccc}
\hline Variável & $n(\%)$ & $\mathrm{AI}$ & $\mathrm{AF}$ & $\mathrm{Ex}$ & $\mathrm{VC}$ \\
& & $M(D P)$ & $M(D P)$ & $M(D P)$ & $M(D P)$ \\
\hline Estatuto & & & & & \\
& & & & & \\
1 (alto) & 30 & 26.24 & 19.2 & 13.14 & 6.62 \\
& $(15.7)$ & $(5.34)$ & $(4.31)$ & $(3.67)$ & $(2.82)$ \\
2 & 15 & 26.81 & 17.47 & 15.73 & 5.53 \\
& $(8.5)$ & $(5.24)$ & $(4-76)$ & $(4.41)$ & $(2.17)$ \\
3 & 112 & 24.93 & 17.77 & 14.39 & 6.31 \\
& $(58.6)$ & $(7.44)$ & $(4.07)$ & $(4.64)$ & $(3.03)$ \\
4 (baixo) & 34 & 25.45 & 17.85 & 16.06 & 7.20 \\
& $(17.8)$ & $(7.04)$ & $(4.63)$ & $(5.12)$ & $(2.90)$ \\
Prestígio & & & & & \\
Alto & 37 & 26.03 & 18,73 & 14.36 & 6.57 \\
& $(15.2)$ & $(6.93)$ & $(4.61)$ & $(5.28)$ & $(2.74)$ \\
Baixo & 205 & 25.24 & 17.66 & 14.9 & 6.64 \\
& $(84.7)$ & $(7.07)$ & $(4.26)$ & $(4.65)$ & $(2.9)$ \\
\hline
\end{tabular}

$\mathrm{AI}=$ Apoio Informacional, $\mathrm{AF}=$ Apoio Financeiro,

$\mathrm{Ex}=$ Expectativas, $\mathrm{VC}=$ Valores e Crenças

Em relação ao estatuto socioeconómico do estudante, o nível médio-alto apresenta níveis mais elevados de apoio informacional, o nível alto apresenta níveis mais elevados de apoio financeiro, e o nível baixo apresenta expectativas mais elevadas, assim como valores e crenças. Em relação ao prestígio do curso, quanto mais alto, mais elevado o apoio informacional e financeiro, mas mais baixas as expectativas e os valores e crenças. 


\section{Discussão e conclusão}

Este estudo visou analisar a influência familiar nas decisões de carreira em relação a diferentes fatores estruturais e processuais familiares. Importa referir que, em termos descritivos, verificou-se que as famílias são, em geral nucleares, e ambos os pais tendem a trabalhar. Este fato aliado às frequências do estatuto

socioeconómico levam a crer que há um perfil relativamente diferente da média geral da população brasileira (Instituto Brasileiro de Geografia e Estatística, 2015). É interessante notar a grande concentração de primogénitos na amostra, nesse sentido, para o dado ser melhor compreendido deve ser observado em conjunto com o número de irmãos.

De um modo geral, os resultados deste estudo vão ao encontro dos obtidos em estudos prévios na área. Em termos de variáveis estruturais, por exemplo, no que respeita o nível socioeconómico (NSE) familiar, quando este é elevado, pode influenciar a realização académica e a posterior escolha por cursos/profissões considerados mais atípicos (e.g., Astin, 1993; Simpson, 2001). A razão porque isto ocorre não é clara, mas alguns autores antecipam que um NSE mais elevado está associado a um conhecimento mais diversificados das profissões, e também a uma procura de profissões consideradas mais prestigiadas (Fisher \& Padmawidjaja, 1999). No que se refere à situação profissional dos pais, em algumas culturas existe uma forte expectativa de que os jovens deem continuidade ao legado familiar, prosseguindo a mesma profissão (Flint, 1993; Tang, Fouad \& Smith, 1999) e, neste sentido, os jovens são estimulados a prosseguir ocupações tradicionais, que são consideradas mais financeiramente estáveis.

Por sua vez, e em termos de variáveis processuais, estudos neste âmbito têm demonstrado o apoio e encorajamento parental tem efeitos nas crenças de autoeficácia (Gushue \& Whitson, 2006) e tomada de decisão acerca do ingresso, adiamento da decisão de desistência, e desistência do curso do ensino superior (Alliman-Brissett, Turner \& Skovholt, 2004; Slaten, \& Baskin, 2014). Também parecem existir diferenças entre o apoio maternal e paternal, sendo o primeiro mais procurado pelos jovens adultos (Guerra \& Braungart-Rieker, 1999). No que respeita as expectativas parentais, se estas forem congruentes com as decisões efetivas dos filhos acerca dos cursos a frequentar, existe uma maior tendência a uma integração académica mais positiva (Bardagi, 2007; Tang, Fouad \& Smith, 1999). Em relação à comunicação e realização de atividades focadas na temática da carreira, apesar de se salientarem a sua pertinência, os estudos demonstram a quase inexistência de conversas sistemáticas e de incentivos à exploração, que favoreçam a partilha de informação e experiências entre pais e filhos (Bardagi, 2007).

As diferenças observadas entre as médias nas escalas relativas à influência familiar não podem ser foco de inferências, mas dado o caráter exploratório do estudo, servem como guia em futuras análises.

\section{Referências}

Alliman-Brissett, A. E., Turner, S. L., \& Skovholt, T. M. (2004). Parent support and African-American adolescents' career self-efficacy. Professional School Counseling, 7, 124-132.

Araújo, A. Taveira, M.C., Mota, A. I., Oliveira, I., Pinto, J. C., Viamonte, I., \& Faria, L. (2012).Parental Participation in Adolescents' Educational and Career Experiences and Planning: A Study with Portuguese Parents. Actas da 6th International Technology, Education, and Development Conference, 5 a 7 Março, Valença, Espanha. https://library.iated.org/view/ARAUJO2012PAR

Astin, A. W. (1993). What matters in college?: Four critical years revisited (Vol. 1). San Francisco: Jossey-Bass.

Bardagi, M. P. (2007). Evasão e comportamento vocacional de universitários: Estudos sobre o desenvolvimento de carreira na graduação. Tese de doutoramento. Universidade Federal do Rio Grande do Sul

Bryant, B. K., Zvonkovic, A. M., \& Reynolds, P. (2006). Parenting in relation to child and adolescent vocational development. Journal of Vocational Behavior, 69(1), 149-175.

Chickering, A. W. (1969). Education and identity. Jossey-Bass.

Dietrich, J., \& Kracke, B. (2009). Career-specific parental behaviors in adolescents' development. Journal of Vocational Behavior, 75, 109-119

Faria, L., Pinto, J. C., \& Taveira, M. D. C. (2007, October). Família e aconselhamento parental: trajectórias de carreira saudáveis. Actas do II Congresso Família, Saúde e Doença: Modelos, Investigação e Prática em Diversos Contextos de Saúde.

Fisher, T. A., \& Padmawidjaja, I. (1999). Parental influences on career development perceived by African American and Mexican American college students. Journal of Multicultural Counseling and Development, 27(3), 136.

Flint, T. A. (1993). Parental and planning influences on the formation of student college choice sets. Research in Higher Education, 33(6), 689-708.

Fouad, N.A., Cotter, E.W, Fitzpatrick, M.E., Kantamneni, N., Carter, L., \& Bernfeld, S. (2010). Development and Validation of the Family Influence Scale. Journal of Career Assessment, 18 (3), 276-291.

Guerra, A. L., \& Braungart Rieker, J. M. (1999). Predicting career indecision in college students: The roles of identity formation and parental relationship factors. The career development quarterly, 47(3), 255-266.

Gushue, G. V., \& Whitson, M. L. (2006). The relationship among support, ethnic identity, career decision selfefficacy, and outcome expectations in African American high school students applying social cognitive career theory. Journal of Career Development, 33, 112-124. 
Hollingshead, A. A. (1975). Four-factor index of social status. Manuscrito não publicado, Yale University, New Haven, CT.

Instituto Brasileiro de Geografia e Estatística (IBGE). (2015). Síntese de indicadores sociais: uma análise das condições de vida da população brasileira 2015. Rio de Janiero: IBGE Retirado de: http://biblioteca.ibge.gov.br/visualizacao/livros/liv950 11.pdf

Lankard, B. A. (1995). Family role in career development. ERIC Clearinghouse.

Lent, R. W., Brown, S. D., Talleyrand, R., McPartland, E. B., Davis, T., Chopra, S. B., ... \& Chai, C. M. (2002). Career choice barriers, supports, and coping strategies: College students' experiences. Journal of Vocational Behavior, 60(1), 61-72.

Otto, L. B. (2000). Youth perspectives on parental career influence. Journal of Career Development, 27(2), 111-118.

Paa, H. K. \& McWhirter, E. H. (2000). Perceived Influences on High School Students' Current Career Expectations. The Career Development Quarterly, 49, 29-44. doi:10.1002/j.2161-0045.2000.tb00749.x

Pachane, G. G., Mercuri, E., \& Polydoro, S. A. J. (2003). A experiência universitária e sua contribuição ao desenvolvimento pessoal do aluno. Estudante universitário: Características e experiências de formação, 155-186.

Pinto, H. R., \& Soares, M. C. (2000). Influência parental no desenvolvimento vocacional. Relatório de investigação. Lisboa: Instituto de Orientação Profissional.

Pinto, H. R., \& Soares, M. C. (2001). Influência parental na carreira: Evolução de perspectivas na teoria, na investigação e na prática. Psychologica, 26, 135-149.

Porto, J. B., \& Tamayo, A. (2006). Influência dos valores laborais dos pais sobre os valores laborais dos filhos. Psicologia: Reflexão \& Crítica, 19(1), 151-158.

Ribeiro, M. A. (2005). O projeto profissional familiar como determinante da evasão universitária: um estudo preliminar. Revista Brasileira de Orientação Profissional, 6(2), 55-70.

Schulenberg, J. E., Vondracek, F. W., \& Crouter, A. (1984). The influence of the family on vocational development. Journal of Marriage and the Family, 46, 129-143. doi:10.2307/351871

Schultheiss, D. (2007). Career development in the context of children's and adolescents' relationships. In V.B. Skorikov \& W. Patton (Eds.), Career development in childhood and adolescence (pp. 169-180). Rotterdam: Sense Publishers.

Simpson, J. C. (2001). Segregated di erences by subject: Racial di erences in the factors influencing academic major between european americans, asian americans, and african, hispanic, and native americans. Journal of Higher Education 72(1), 63-100.

Slaten, C. D., \& Baskin, T. W. (2014). Examining the impact of peer and family belongingness on the career decision-making difficulties of young adults: A path analytic approach. Journal of Career Assessment, 22(1), 59-74.

Super, D. E. (1976). Career education and the meanings of work. US Department of Health, Education, and Welfare, Office of Education: for sale by the Supt. of Docs., US Govt. Print. Off.

Tang, M., Fouad, N. A., \& Smith, P. L. (1999). Asian Americans' career choices: A path model to examine factors influencing their career choices. Journal of Vocational Behavior, 54(1), 142-157.

Whiston, S. \& Keller, B. (2004). The influences of the family of origin on career development: A review and analysis. The Counselling Psychologist, 32, 493-568. doi: $10.1177 / 0011000004265660$. 